\title{
Microfluidic Tools Reducing Sample Amount in Serial Crystallography with XFELs
}

\author{
Alexandra Ros \\ Arizona State University, Tempe, United States of America \\ alexandra.ros@asu.edu
}

The recent advances of X-ray free electron lasers (XFEL) have enabled serial femtosecond crystallography (SFX) and structure determination for complex proteins such as membrane proteins in high resolution. ${ }^{1-3}$ Importantly, time-resolved (TR) studies have emerged allowing to assess their reaction dynamics. Initial demonstrations focused on light induced reactions; however, a large class of biological macromolecules acts by reaction with specific substrates requiring fast mixing approaches for TR-studies. Microfluidic tools in combination with common liquid injectors for protein crystals allow mixing times in the millisecond to second range, which is suitable to study the dynamics of enzymatic reactions with SFX at XFELs. A large drawback for TR-SFX with substrate-initiated reactions remains the large amount of protein and crystals needed to study the time evolution of a reaction. Every time point to be assessed requires a full data set which multiplies the amount of protein crystals needed by the number of time points to be studied. This may result in unsurmountable protein sample limitations requiring hundreds of mg of protein, which are not attainable for many proteins. Microfluidics allows to tackle this issue by reducing the required amount of protein sample. We propose to inject protein crystals with segmented flow approaches, which deliver crystals to the XFEL only when it pulses. We demonstrate how protein crystals in their mother liquor can be encapsulated in droplets surrounded by an immiscible oil and how these droplets can be intersected with an XFEL using common liquid jet injection methods. We demonstrated this approach reducing the amount of sample required to solve the room temperature structure of 3-deoxy-D-manno-2-octulosonate-8-phosphate synthase (KDO8PS) at the SPB/SFX instrument at the EuXFEL. ${ }^{4}$ Furthermore, we demonstrated the ability to electrically trigger the crystal laden droplet release in the microfluidic droplet generator, the interfacing of this approach with miniaturized optical droplet detection and an electronic feedback mechanism to tune the droplet release at a desired frequency matching the repetition rate of a particular XFEL instrument. This approach has been recently tested at the Macromolecular Femtosecond Crystallography instrument at the Linac Coherent Light Source, where the feedback mechanism was successfully implemented. Diffraction was recorded for lysozyme and the protein KDO8PS and the ability to tune the droplet release with a desired delay to the XFEL reference signal was also achieved. This is important to optimize the synchronization with the XFEL when implemented in particular chambers and various geometrical realizations of the droplet generator in relation to the XFEL interaction spot. In follow up experiments, we will assess the amount of sample that is required to obtain a full data set for KDO8PS and couple this strategy with microfluidic mixers, which have already been integrated into the 3D-printed droplet generators. With this approach, we predict that the amount of protein required to achieve a full data set can be reduced by nearly $90 \%$.

1. Spence, J. C. H.; Weierstall, U.; Chapman, H. N. Reports on Progress in Physics 2012, 75.

2. Chapman, H. N.; Fromme, P.; Barty, A., et al. Nature 2011, 470, 73-U81.

3. Martin-Garcia, J. M.; Conrad, C. E.; Coe, J., et al. Archives of Biochemistry and Biophysics 2016, 602, 32-47.

4. $\quad$ Echelmeier, A.; Villarreal, J. C.; Kim, D., et al. Nat Comm 2020, 4511.

Keywords: Microfluidics, droplets, synchronization, sample delivery 